\title{
INNER FUNCTIONS AND CYCLIC VECTORS IN THE BLOCH SPACE
}

\author{
J. M. ANDERSON, J. L. FERNÁNDEZ AND A. L. SHIELDS
}

\begin{abstract}
In this paper we construct a singular inner function whose polynomial multiples are dense in the little Bloch space $\mathscr{B}_{0}$. To do this we construct a singular measure on the unit circle with "best possible" control of both the first and second differences.
\end{abstract}

\section{BACKGROUND}

The Bloch space $\mathscr{B}$ in the open unit disc $\mathbb{D}$ in the complex plane is the space of all those analytic functions $f$ such that $\left(1-|z|^{2}\right) f^{\prime}(z)$ is bounded in $\mathbb{D}$. We norm $\mathscr{B}$ as follows:

$$
\|f\|=|f(0)|+\sup \left\{\left(1-|z|^{2}\right)\left|f^{\prime}(z)\right|: z \in \mathbb{D}\right\} .
$$

With this norm $\mathscr{B}$ is a Banach space and $\mathscr{B}_{0}$ a closed subspace. Here $\mathscr{B}_{0}$, sometimes called the "little" Bloch space, denotes the set of those $f$ in $\mathscr{B}$ for which $\left(1-|z|^{2}\right) f^{\prime}(z) \rightarrow 0$ as $|z| \uparrow 1$. We recall several results about these spaces (see [ACP74 and An85] for details).

Let $L_{a}^{1}$ denote the set of analytic functions that are in $L^{1}$ with respect to area measure in $\mathbb{D}$ and let $\mathscr{J}$ denote the set of analytic functions $g$ in $\mathbb{D}$ for which $g^{\prime} \in L_{a}^{1}$, with the norm

$$
\|g\|=|g(0)|+\frac{1}{\pi} \int_{\mathbb{D}}\left|g^{\prime}\right| d A .
$$

Here $d A$ denotes area measure. If $f \in \mathscr{B}$ and $g \in \mathscr{J}$ have the power series $f=\sum \hat{f}(n) z^{n}, g=\sum \hat{g}(n) z^{n}$, and if $G(z)=\left[(z g)^{\prime}-g(0)\right] / z$, then the following limit exists:

$$
\begin{aligned}
\langle f, g\rangle & =\lim _{r \uparrow 1} \sum_{0}^{\infty} \hat{f}(n) \hat{g}(n) r^{n} \\
& =\hat{f}(0) \hat{g}(0)+\frac{1}{\pi} \iint f^{\prime}\left(r e^{i \vartheta}\right) G\left(r e^{-i \vartheta}\right)\left(1-r^{2}\right) r d r d \vartheta .
\end{aligned}
$$

Received by the editors August 15, 1988 and, in revised form, February 1, 1989.

1980 Mathematics Subject Classification (1985 Revision). Primary 46E15; Secondary 30H05, $46 \mathrm{~J} 15$.

This research was supported in part by the National Science Foundation. 
Using (3), each $g \in \mathscr{I}$ defines a bounded linear functional on $\mathscr{B}_{0}$, and every bounded linear functional has this form. Likewise, each $f \in \mathscr{B}$ defines a bounded linear functional on $\mathscr{I}$, and every bounded linear functional has this form. Furthermore, the linear functional norm for $g$ is equivalent to the norm (2), and for $f$ it is equivalent to (1). Thus we may say that

$$
\mathscr{B}_{0}^{*}=\mathscr{I}, \quad \mathscr{I}^{*}=\mathscr{B},
$$

in the sense of isomorphic Banach spaces. (Note, however, that $\mathscr{B}$ with the norm (1) is actually isometric to $\mathscr{B}_{0}^{* *}$, see [RS70].)

Further, the polynomials are norm dense in $\mathscr{B}_{0}$ and in $\mathscr{I}$, and are weak* dense in $\mathscr{B}$ ( $\mathscr{B}$ is not separable). Also, $\mathscr{B}_{0}, \mathscr{I}$, and $\mathscr{B}$ are isomorphic, as Banach spaces, to the sequence spaces $c_{0}, l^{1}$, and $l^{\infty}$ (it is sufficient to establish this result for $\mathscr{B}_{0}$, and this can be deduced from Theorem 7 of [SW78]).

Lemma 1. (a) If $\left\{f_{n}\right\} \subset \mathscr{B}$ then $f_{n} \rightarrow 0$ weak ${ }^{*}$ if and only if $f_{n}(z) \rightarrow 0$ for all $z$ in $\mathbb{D}$, and $\sup \left\|f_{n}\right\|<\infty$.

(b) If $\left\{f_{\alpha}\right\} \subset \mathscr{B}, 0 \leq \alpha<1$, then $\lim f_{\alpha} \rightarrow 0$ (as $\alpha \uparrow 1$ ) weak ${ }^{*}$ if and only if $\lim f_{\alpha}(z) \rightarrow 0(\alpha \uparrow 1)$ for all $z \in \mathbb{D}$, and lim- sup $\left\|f_{\alpha}\right\|<\infty$.

Remark. (a) and (b) remain valid if $\mathscr{B}$ is replaced by $\mathscr{B}_{0}$ and weak* is replaced by weakly.

Proof. Part (a) follows from the dominated convergence theorem, and (b) follows from (a) by contradiction; we omit the details.

We may derive a growth estimate for Bloch functions from (1). Since $f(z)=$ $f(0)+\int f^{\prime}$ (integrating along the radius), we have

$$
\begin{aligned}
|f(z)| & \leq|f(0)|+\|f\| \int_{0}^{r}\left(1-t^{2}\right)^{-1} d t \leq\left\{1+\frac{1}{2} \log \frac{1+r}{1-r}\right\}\|f\| \\
& \leq\left\{1+\log \frac{1}{1-r}\right\}\|f\| .
\end{aligned}
$$

Thus $\mathscr{B}$ is contained in $L_{a}^{p}$ (the analytic $L^{p}$ functions in $\mathbb{D}$ ), for all $p<\infty$. For the Hardy spaces we have $H^{\infty} \subset \mathscr{B}$, but $H^{p}$ is not contained in $\mathscr{B}$ for any $p<\infty$; also, $\mathscr{B}$ is not contained in the Nevanlinna class.

In this paper we study (weak*) cyclic vectors for the space $\mathscr{B}$. These are the functions $f$ in $\mathscr{B}$ whose polynomial multiples are weak* dense in $\mathscr{B}$ (that is, they are cyclic vectors in the weak* topology for the operator of multiplication by $z$ on $\mathscr{B}$ ). We note that if $f$ is in $\mathscr{B}_{0}$, then $f$ is (norm) cyclic in $\mathscr{B}_{0}$ if and only if it is weak* cyclic in $\mathscr{B}$. Indeed, if $\mathscr{P}$ denotes the polynomials, then the linear manifold $\mathscr{P} f$ is norm dense in $\mathscr{B}_{0}$ if and only if no linear functional annihilates it; this is precisely the condition for weak* density in $\mathscr{B}$. When we refer to cyclic vectors in $\mathscr{B}$ the weak* topology is always understood.

We note that if $f$ is cyclic in $\mathscr{B}$, then $f$ has no zeros in $\mathbb{D}$ (since point evaluations are weak* continuous functionals on $\mathscr{B}$ ). This condition is not sufficient, however, as indicated below. A sufficient condition was given in 
[ACP74, p. 28]: if $|f(z)| \geq c>0$, then $f$ is cyclic in $\mathscr{B}$. Theorem 1 below is an extension of this result.

Since $H^{\infty} \subset \mathscr{B}$ it is natural to begin by asking which $H^{\infty}$ functions are cyclic in $\mathscr{B}$. The corollary to the next result gives a partial answer. We recall that the weak* topology on $H^{\infty}$ results from the pairing of $H^{\infty}$ with the quotient space $L_{+}^{1}$ (where $L_{+}^{1}=\left\{\sum_{0}^{\infty} \hat{\varphi}(n) z^{n}: \varphi \in L^{1}\right\}$, with the quotient norm); this pairing can be expressed by (3). The next result is the "predual" form of the containment of $H^{\infty}$ in $\mathscr{B}$; in this form the proof is considerably more difficult.

Proposition 1. $\mathscr{I} \subset L_{+}^{1}$.

Proof. Let $g \in \mathscr{I}$; we must show that there exists $\varphi \in L^{1}$ such that $\hat{g}(n)=$ $\hat{\varphi}(n) \quad(n \geq 0)$. We know that

$$
\lim _{r \rightarrow 1} \sum_{0}^{\infty} \hat{f}(n) \hat{g}(n) r^{n}
$$

exists for all $f \in \mathscr{B}$, and hence for all $f \in H^{\infty}$. The principal theorem in [PSW67] now asserts the existence of $\varphi$.

Corollary. If $f \in H^{\infty}$ is outer, then $f$ is cyclic in $\mathscr{B}$.

Proof. We recall that the analogue of Beurling's theorem for $H^{2}$ is valid for $H^{\infty}$ in the weak* topology (see [RS66, Theorem 5.5] for example); in particular, the polynomial multiplies $\mathscr{P} h$ of a function $h \in H^{\infty}$ are weak* dense in $H^{\infty}$ if and only if $h$ is an outer function. Thus no element of $L_{+}^{1}$ annihilates $\mathscr{P} f$; by the proposition, no element of $\mathscr{I}$ annihilates $\mathscr{P} f$, and so $f$ is (weak*) cyclic in $\mathscr{B}$.

What about singular inner functions? Since $\mathscr{B} \subset L_{a}^{2}$ it can be shown that if a function (in particular, a singular inner function) is cyclic in $\mathscr{B}$ then it is cyclic in $L_{a}^{2}$. (The proof is similar to the proof of the corollary above. The dual of $L_{a}^{2}$ in the pairing (3) is the space of analytic functions whose first derivative is in $L_{a}^{2}$, that is, the Dirichlet space. The containment corresponding to Proposition 1 is trivial since $L^{2} \subset L^{1}$.)

One has a complete description of the singular inner functions that are cyclic in $L_{a}^{2}$ from Korenblum [Ko81] and Roberts [R85] (see also Joel Shapiro's notes [jS80]). Namely, the singular measure must put no mass on any closed subset of $\partial \mathbb{D}$ that is "thin" in the sense of Beurling, Carleson, and Hayman (see [BS84, p. 274] for these references and for the definition of the sets). See [S85] for more information about cyclicity in $L_{a}^{2}$. In particular, the singular inner function $\exp \{(z+1) /(z-1)\}$ is not cyclic in $L_{a}^{2}$, (H. S. Shapiro has informed us that this result is due to M. V. Keldyš), and thus is an example of a function with no zeros that is not cyclic in $\mathscr{B}$.

The purpose of this paper is to exhibit singular inner functions that are cyclic in $\mathscr{B}$; our examples will actually lie in $\mathscr{B}_{0}$ and thus are cyclic there. 
We wish to thank Professor Leon Brown for helpful discussions about some of the material in this paper.

\section{A SUFFICIENT CONDITION FOR CYCLICITY}

Theorem 1. Let $\varphi(t)$ be a continuous, nondecreasing function on $[0,1]$. Let $f$ be analytic in $\mathbb{D}$. If there exist $a, b>0$ such that for all $z \in \mathbb{D}$ we have

$$
\begin{aligned}
& |f(z)| \geq a \varphi(1-r) \quad(r=|z|), \\
& \left|f^{\prime}(z)\right| \leq \frac{b}{1-r} \varphi(1-r) \quad(r=|z|),
\end{aligned}
$$

then $f$ is cyclic in $\mathscr{B}$.

Rem. Note that $f \in \mathscr{B}$ by (6). The proof below is modelled on the proof of Theorem 3.8 in [ACP74].

Proof. For $0 \leq t<1$ we have (with $r=|z|, s=|w|$ ),

$$
\begin{aligned}
|f(z)-f(t z)| & =\left|\int_{t z}^{z} f^{\prime}(w) d w\right| \leq b\|f\| \varphi(1-t r) \int_{t r}^{r}(1-s)^{-1} d s \\
& =b\|f\| \varphi(1-t r) \log \left\{\frac{1-t r}{1-r}\right\} .
\end{aligned}
$$

Let $[f]$ denote the weak* closure in $\mathscr{B}$ of the polynomial multiples of $f$; thus our goal is to show that $[f]=\mathscr{B}$.

Let $f_{t}(z)=f(t z), 0 \leq t<1$. Since $1 / f_{t}$ is analytic on the closed unit disc one shows that $\left(1 / f_{t}\right) f \in[f]$. Also, $f(z) / f_{t}(z) \rightarrow 1$ as $t \uparrow 1$. Thus, by Lemma 1 , to complete the proof we need only show that $\left\|f / f_{t}\right\|$ is bounded. From (1), since $\left(f / f_{t}\right)(0)=1$, it remains to show that $(1-r)\left|\left(f / f_{t}\right)^{\prime}(z)\right|$ is bounded in $\mathbb{D}(r=|z|)$; equivalently, that $(1-r)\left|\left\{\left(f-f_{t}\right) / f_{t}\right\}^{\prime}(z)\right|$ is bounded. We have

$$
\left(\frac{f-f_{t}}{f_{t}}\right)^{\prime}=\frac{\left(f-f_{t}\right)^{\prime}}{f_{t}}-\frac{\left(f-f_{t}\right)\left(f_{t}\right)^{\prime}}{f_{t}^{2}}=F_{1}+F_{2} \text {. }
$$

Let $c$ denote a constant, not necessarily the same each time it occurs. Since $\left(f_{t}\right)^{\prime}(z)=t f^{\prime}(t z)$, from $(5),(6)$, and (7) we have:

$$
\begin{aligned}
& \left|F_{1}(z)\right| \leq b\left\{\frac{\varphi(1-r)}{1-r}+t \frac{\varphi(1-t r)}{1-t r}\right\} / a \varphi(1-t r) \leq \frac{c}{1-r}, \\
& \mid F_{2}(z) \leq c\|f\|\left(\log \frac{1-t r}{1-r}\right) /(1-t r) .
\end{aligned}
$$

Since $(\log x) / x$ is bounded for $1 \leq x<\infty$ we see that $(1-r) F_{2}(z)$ is bounded in $\mathbb{D}$; also, $(1-r) F_{1}(z)$ is bounded, which completes the proof.

Problem 1. If $\varphi(t)=1 / \log (e / t)$, can condition (6) be omitted in the statement of Theorem 1 ?

An affirmative answer would, by (4), imply an affirmative answer to the following question (which is open even when $f$ is bounded).

Problem 2. If $f$ and $1 / f$ are both in $\mathscr{B}$, then must $f$ be cyclic in $\mathscr{B}$ ? 


\section{SMOOTHNESS OF INNER FUNCTIONS}

Let $\mu$ be a positive finite Borel measure on $\mathbb{T}=\partial \mathbb{D}$. By the modulus of continuity and the modulus of smoothness of $\mu$ we mean the following two functions:

$$
\begin{aligned}
& \omega_{1}(\delta ; \mu)=\sup \{\mu(I): m(I) \leq \delta, I \text { a subarc of } \mathbb{T}\}, \\
& \omega_{2}(\delta ; \mu)=\sup \{|\mu(I)-\mu(J)|: m(I)=m(J) \leq \delta, I, J \text { contiguous arcs }\} .
\end{aligned}
$$

Here $m$ denotes normalized Lebesgue measure on $\mathbb{T}$. Thus in the definition of $\omega_{2}, I$ and $J$ can be arcs of the form:

$$
I=\left\{e^{i t}: s \leq t<s+h\right\}, \quad J=\left\{e^{i t}: s-h \leq t<s\right\}, \quad 0<h \leq 2 \pi \delta .
$$

We only consider continuous measures (i.e., points have measure 0 ), Thus the endpoints of the arcs will not matter. If either $\omega_{1}$ or $\omega_{2}$ tends to 0 as $\delta$ tends to 0 , then the measure $\mu$ is continuous; if $\mu$ is continuous then both $\omega_{1}$ and $\omega_{2}$ tend to 0 . A variant of the next result is in [hS64] (see also $\S 6$ of [jS80]). We write $\omega_{1}(\delta)$ instead of $\omega_{1}(\delta ; \mu)$.

Theorem 2. Let $S_{\mu}$ be a singular inner function with singular measure $\mu$. Then

$$
\left|S_{\mu}(z)\right| \geq \exp \left\{-6 \frac{\omega_{1}(1-r)}{1-r}\right\}, \quad r=|z| .
$$

Before giving the proof we require two lemmas.

Lemma 2. Let $|t| \leq \pi, \delta=1-r, 0 \leq r<1$. Then

$$
\frac{1-r^{2}}{\left|r-e^{i t}\right|^{2}} \leq \frac{2 \delta}{\delta^{2}+(t / \pi)^{2}} \text {. }
$$

Proof. The inequality is equivalent to

$$
\begin{aligned}
(1+r)\left\{\delta^{2}+(t / \pi)^{2}\right\} & \leq 2\left\{1-2 r \cos t+r^{2}\right\} \\
& =2\left\{\delta^{2}+2 r(1-\cos t)\right\} .
\end{aligned}
$$

This inequality in turn is equivalent to

$$
(1+r)(t / \pi)^{2} \leq \delta^{3}+4 r(1-\cos t) .
$$

It will be sufficient to prove this when $0 \leq t \leq \pi$.

Case 1. $\frac{\pi}{2} \leq t \leq \pi$. Since the cosine is negative and the left side of $(10)$ is largest when $t=\pi$, it is sufficient to show that $1+r \leq \delta^{3}+4 r$; this follows by multiplying out and comparing terms.

Case 2. $0 \leq t \leq \frac{\pi}{2}$. The function $(\sin t) / t$ is decreasing, hence so is the function $(1-\cos t) / t^{2}=2\left(\sin \frac{t}{2}\right)^{2} / t^{2}$. Thus $1-\cos t \geq 4(t / \pi)^{2}$ for $0 \leq t \leq \frac{\pi}{2}$. Substituting this in the right side of $(10)$ and simplifying we see that it will be sufficient to prove that

$$
\delta^{3}+(15 r-1)(t / \pi)^{2} \geq 0
$$


This is obvious if $15 r \geq 1$. If $15 r<1$, then the worst case is when $t=\pi / 2$. Thus we must show that $4 \delta^{3}>1-15 r$, and this follows by multiplying out and comparing terms.

If $\mu$ is a Borel measure on $\mathbb{T}$, then $P[\mu](z)$ denotes the Poisson integral of $\mu$, evaluated at the point $z \in \mathbb{D}$.

Lemma 3. If $\mu$ is a positive finite Borel measure on $\mathbb{T}$, then

$$
P[\mu](z) \leq 6 \frac{\omega_{1}(1-r)}{1-r}, \quad r=|z| .
$$

Proof. Let $\delta=1-r$, let $N$ be the largest integer such that $2 \delta N \leq 1$, and let

$$
\begin{gathered}
I_{k}=\left\{e^{i t}: 2 \pi \delta k \leq t<2 \pi \delta(k+1)\right\}, \quad k=0,1, \ldots, N-1, \\
I_{N}=\left\{e^{i t}: 2 \pi \delta N \leq t \leq \pi\right\} .
\end{gathered}
$$

Thus $m\left(I_{k}\right)=\delta, k<N$, and $m\left(I_{N}\right)<\delta$, where, as above, $m$ denotes the normalized Lebesgue measure on $\mathbb{T}$. Thus $\mu\left(I_{k}\right) \leq \omega_{1}(\delta), k \leq N$. We break the Poisson integral into two parts: $0 \leq t \leq \pi$, and $-\pi \leq t \leq 0$. By Lemma 2 we have

$$
\begin{aligned}
\int_{0}^{\pi} \frac{1-r^{2}}{\left|r-e^{i t}\right|^{2}} d \mu(t) & \leq \sum_{0}^{N} \int_{I_{k}} \frac{2 \delta}{\delta^{2}+(t / \pi)^{2}} d \mu \leq 2 \omega_{1}(\delta) \sum_{0}^{N} \frac{1}{\delta+4 \delta k^{2}} \\
& \leq 3 \frac{\omega_{1}(\delta)}{\delta} .
\end{aligned}
$$

Here we have used the fact that $\pi^{2}<10$ and therefore

$$
\sum_{0}^{\infty} \frac{1}{1+4 k^{2}}<1+\frac{1}{5}+\sum_{2}^{\infty} \frac{1}{4 k^{2}}=1.2+\frac{1}{4}\left(\frac{\pi^{2}}{6}-1\right)<1.5 .
$$

A similar estimate for the other integral completes the proof.

Proof of Theorem 2. Since $\left|S_{\mu}(z)\right|=\exp \{-P[\mu](z)\}$, the result follows from Lemma 3

We say that a positive continuous function $\varphi$ on the half open interval $(0,1]$ satisfies conditions $(*)$ if the following holds.

(*) There exist constants $c$, $a$ with $c>0$ and $0<a<1$, such that

$$
\frac{\varphi(x)}{x^{a}} \geq c \frac{\varphi(y)}{y^{a}}
$$

for all $x, y$ with $0<x \leq y \leq 1$.

For example, if $\varphi(x)=x^{\alpha}\{\log (e / x)\}^{-\beta}$ for some $\alpha, 0 \leq \alpha<1$, and some $\beta \geq 0$, then $\varphi$ satisfies condition $(*)$. We shall be interested in the case $\alpha=0, \beta=\frac{1}{2}$. In the terminology of $\mathrm{S}$. $\mathrm{N}$. Bernstein (see the paragraph preceding Theorem 3 in [Be49]) the function $\varphi(x) / x^{a}$ is almost decreasing on the interval $(0,1]$. 
The following lemma will give us an upper bound for the derivative of a singular inner function in terms of the second difference. It is suggested by the proof of Theorem 1 of [DSS66] (which corresponds to taking $\varphi=1$ ). However we have organized the details of the proof somewhat differently because the proof in [DSS66] used two facts that are not available here: the self-conjugacy of the Zygmund class $\Lambda^{*}$, and the equivalence between $\Lambda^{*}$ and the growth of the second derivative.

Lemma 4. Let $\mu$ be a positive, finite Borel measure on $\mathbb{T}$, and let

$$
F(z)=\int \frac{w+z}{w-z} d \mu(w), \quad w=e^{i t}
$$

Let $\varphi(t)$ be positive, continuous, nondecreasing on the half open interval $(0,1]$, and let $\varphi$ satisfy condition $(*)$ above. If $\omega_{2}(\delta ; \mu)=O(\delta \varphi(\delta))$, then

$$
\left|F^{\prime}(z)\right|=O\left(\frac{\varphi(1-r)}{1-r}\right), \quad r=|z| .
$$

Proof. In this proof $c$ will denote a constant, not necessarily the same at each occurrence. We assume that $\|\mu\|=1$. Let $\nu=\mu-m$, where $m$ denotes normalized Lebesgue measure on $\mathbb{T}$. Then $\hat{\nu}(0)=0$ and $\hat{\nu}(n)=\hat{\mu}(n)$ for $n \neq 0$. Also, $\omega_{2}(\delta ; \nu)=\omega_{2}(\delta ; \mu)$. Let $g(t)=\nu\left(I_{t}\right)$, where

$$
I_{t}=\left\{e^{i \vartheta}: 0 \leq \vartheta<t\right\}, \quad 0 \leq t \leq 2 \pi .
$$

Then $g$ is continuous (points have measure 0 since $\omega_{2}(\delta)$ tends to 0 as $\delta$ tends to 0 ); also, $g(0)=g(2 \pi)=0$. We extend $g$ to a periodic function on the real line. Integrating by parts we have

$$
2 \pi i n \hat{g}(n)=i n \int_{0}^{2 \pi} e^{-i n t} g(t) d t=\int e^{-i n t} d \nu=\hat{\mu}(n), \quad n \neq 0 .
$$

From $(w+z)(w-z)^{-1}=1+2 \sum_{1}^{\infty}(\bar{w} z)^{n}$, and $w z(w-z)^{-2}=\sum_{1}^{\infty} n(\bar{w} z)^{n}$ we have

$$
F(z)-1=2 \sum_{1}^{\infty} \hat{\mu}(n) z^{n}=2 i \int_{-\pi}^{\pi} w z(w-z)^{-2} g(t) d t, \quad w=e^{i t} .
$$

Thus

$$
\begin{aligned}
F^{\prime}(z) & =2 i \int_{-\pi}^{\pi} w(w-z)^{-2} g(t) d t+4 i z \int_{-\pi}^{\pi} w(w-z)^{-3} g(t) d t . \\
& =2 i h_{1}(z)+4 i z h_{2}(z) .
\end{aligned}
$$

Suppose we could show that $\left|h_{2}(z)\right| \leq c \varphi(\delta) / \delta, \delta=1-|z|$. Since $h_{1}^{\prime}=2 h_{2}$, we would have $h_{1} \in \mathscr{B}$; thus $\left|h_{1}\right|=O\left(\log \delta^{-1}\right)$ as $\delta \rightarrow 0$ by (4). This is $O(\varphi(\delta) / \delta)$ since, taking $x=\delta$ and $y=1$ in condition $(*)$, we have $\varphi(\delta) \geq c \delta^{a}$ for some $a<1$. Thus to complete the proof it suffices to estimate $h_{2}$ as above. 
Let $z=r e^{i \vartheta}, \delta=1-r, w=e^{i t}$, and $A(r, t)=w(w-r)^{-3}$. Then

$$
h_{2}(z)=\int_{\vartheta}^{\pi+\vartheta} w(w-z)^{-3} g(t) d t+\int_{-\pi+\vartheta}^{\vartheta} w(w-z)^{-3} g(t) d t \text {. }
$$

Put $s=t-\vartheta$; then, in the second integral, replace $s$ by $-s$. A little calculation shows that, putting $h=e^{2 i \vartheta} h_{2}$, we have

$$
h(z)=\int_{0}^{\pi} A(r, s) g(\vartheta+s) d s+\int_{0}^{\pi} A(r, s)^{*} g(\vartheta-s) d s,
$$

where ${ }^{*}$ denotes complex conjugation. To complete the proof it will be sufficient to show that $|h(z)| \leq c \varphi(\delta) / \delta$ ) (where $\delta=1-|z|$ ).

By a result of [SW82], this is equivalent to showing that $|\operatorname{Re} h|$ satisfies the same estimate. (See Lemma 1(ii), Lemma 2, and Theorem 1 of [SW82]. To use the notation of that paper, let $x=1 / t$, and $\psi(x)=\varphi(t) / t$. Then $\psi(x) / x$ is increasing, and $\psi(x) / x^{1-a}$ is almost decreasing, and so $\psi$ satisfies Conditions (U) and (L) of [SW82].) We have

$$
\begin{aligned}
\operatorname{Re} h(z) & =\int_{0}^{\pi}(\operatorname{Re} A)[g(\vartheta+s)+g(\vartheta-s)] d s \\
& =\int_{0}^{\pi}(\operatorname{Re} A)[g(\vartheta+s)+g(\vartheta-s)-2 g(\vartheta)] d s .
\end{aligned}
$$

To justify the last equality we note that $A(r, s)$ is represented by a power series, in the variable $e^{-i s}$, with real coefficients and no constant term. Thus $\operatorname{Re} A$ is represented by a cosine series with no constant term, and therefore $\int_{0}^{\pi} \operatorname{Re} A=0$.

Taking absolute values and replacing $\operatorname{Re} A$ by $|A|$ we have

$$
|\operatorname{Re} h| \leq \int_{0}^{\pi}\left|e^{i s}-r\right|^{-3} \omega_{2}(s ; \mu) d s \leq c \int_{0}^{\pi}\left|e^{i s}-r\right|^{-3} s \varphi(s) d s .
$$

We may assume that $r>\frac{1}{2}$ and so $\delta<\frac{1}{2}$. Let $n$ denote the largest integer such that $2^{n} \delta<\pi$. Let

$$
\begin{gathered}
I_{0}=\left\{e^{i \vartheta}: 0 \leq \vartheta<\delta\right\}, \quad I_{n+1}=\left\{e^{i \vartheta}: 2^{n} \delta \leq \vartheta \leq \pi\right\}, \\
I_{k}=\left\{e^{i \vartheta}: 2^{k-1} \delta \leq \vartheta<2^{k} \delta\right\}, \quad 1 \leq k \leq n .
\end{gathered}
$$

Then we have

$$
\begin{aligned}
\int_{I_{0}}\left|e^{i s}-r\right|^{-3} s \varphi(s) d s & \leq \delta^{-3} \delta \varphi(\delta) \delta=\varphi(\delta) / \delta \\
\int_{I_{k}}\left|e^{i s}-r\right|^{-3} s \varphi(s) d s & \leq c\left(2^{k} \delta\right)^{-3} 2^{k} \delta \varphi\left(2^{k} \delta\right) 2^{k} \delta \\
& \leq c\left(2^{k} \delta\right)^{-1}\left(2^{k}\right)^{a} \varphi(\delta), \quad 1 \leq k \leq n+1 .
\end{aligned}
$$

To justify the last inequality we note that from condition $(*)$ we have, for $\lambda>1$,

$$
\frac{\varphi(x)}{x^{a}} \geq c \frac{\varphi(\lambda x)}{(\lambda x)^{a}}
$$


and so $\varphi(\lambda x) \leq c \lambda^{a} \varphi(x)$. We now sum over $k$ from 0 to $n+1$; the sum is bounded since $a<1$, and we obtain the desired estimate for $|\operatorname{Re} h|$. This completes the proof of the lemma.

Theorem 3. If $S_{\mu}$ is a singular inner function with singular measure $\mu$, and if $\omega_{2}(\delta ; \mu)=O(\delta \varphi(\delta))$, with $\varphi$ as in Lemma 4 , then

$$
\left|S_{\mu}^{\prime}(z)\right| \leq c \frac{\varphi(1-r)}{1-r}, \quad r=|z| .
$$

Proof. We have $S_{\mu}(z)=\exp \{F(z)\}$, where $F$ is defined by (12). Thus $\left|S_{\mu}^{\prime}(z)\right|$ $=\left|S_{\mu}(z) F^{\prime}(z)\right| \leq\left|F^{\prime}(z)\right|$, and the result follows by Lemma 4 .

Combining Theorems 1,2 , and 3 we see that to construct a singular inner function that is cyclic in $\mathscr{B}$ it is sufficient to construct a singular measure with suitable control of the first and second differences (that is, of the moduli of continuity and of smoothness). Note that in estimating $S_{\mu}^{\prime}$ in the proof of Theorem 3 above we threw away the contribution made by $S_{\mu}$, and put the entire burden on estimating $F^{\prime}$.

\section{CONSTRUCTION OF THE MEASURE}

Let $\mu$ be a positive finite Borel measure on $\mathbb{T}$, and as before let $\omega_{1}$ and $\omega_{2}$ denote the first and second differences of $\mu$ (that is, the moduli of continuity and of smoothness). It is trivial that if $\omega_{1}(t) \leq c t$, then $\mu$ is absolutely continuous with bounded Radon-Nikodým derivative. Also, it is known that if $\int_{0}^{1} t^{-3}\left[\omega_{2}(t)\right]^{2} d t<\infty$, then $\mu$ is absolutely continuous with $L^{2}$ derivative (see [SZ63, Theorem H], for a simple proof see [hS68, p. 274]). In the converse direction the following is known. First, if $\alpha(t)$ is a positive continuous function on $(0,1]$, and if $\alpha(t) \rightarrow \infty$ as $t \rightarrow 0$, then there exists a positive singular measure with $\omega_{1}(t) \leq c t \alpha(t)$ (see [HK37, §5]). Also, if $\beta(t)$ is a continuous increasing function on $[0,1]$, with $\beta(0)=0$ and $\int_{I} t^{-1}[\beta(t)]^{2}=\infty$, then there exists a positive singular measure with $\omega_{2}(t) \leq c t \beta(t)$. (See [hS68, p. 266], or [Ka69, p. 192]. In [Ka69] there is the additional requirement that $2 \beta(t) \geq \beta(4 t)$.)

In [ABB77, Problem 7.32, p. 147] the question was asked as to whether a singular measure existed with both $\omega_{1}(t) \leq c t \alpha(t)$ and $\omega_{2}(t) \leq c t \beta(t)$, as above. The following result shows that this is indeed the case.

We shall identify $\mathbb{T}$ with the closed unit interval $[0,1]$, with 0 and 1 identified.

Theorem 4. Let $\alpha, \beta$ be positive continuous functions on $(0,1]$, such that $\alpha$ is decreasing, $\beta$ is increasing, $\alpha(1) \geq 2, \beta(1) \leq 1$, and

(a) $\alpha(t) \rightarrow \infty, \beta(t) \rightarrow 0,(t \rightarrow 0)$,

(b) $\int_{0}^{1} t^{-1}[\beta(t)]^{2} d t=\infty$,

(c) $2 \beta(t) \geq \beta(4 t), 0 \leq t \leq \frac{1}{4}$. 
Then there exists a singular Borel probability measure $\mu$ on $\mathbb{T}$, with

(1) $\omega_{1}(t ; \mu) \leq 8 t \alpha(t)$,

(2) $\omega_{2}(t ; \mu) \leq 36 t \beta(t)$.

Remark. Let $\beta(t)=\{\log (e / t)\}^{-1 / 2}$ and $\alpha(t)=2-48^{-1} \log \beta(t)$. One verifies that condition (c) above is satisfied. Let $S$ be the singular inner function associated with the measure $\mu$ provided by Theorem 4 . Then by Theorems 2 and 3 we will have:

$$
\left|S^{\prime}(z)\right| \leq c \beta(1-r) /(1-r), \quad|S(z)| \geq e^{-96} \beta(1-r) .
$$

It follows from Theorem 1 that $S$ is cyclic in the little Bloch space $\mathscr{B}_{0}$.

Proof. The construction is modelled on that in [Ka69], where however only the second difference is controlled. The need to control the first difference as well complicates our construction considerably.

As indicated above we identify $\mathbb{T}$ with the interval $[0,1]$, with 0 and 1 identified. Our measure will be constructed as the limit of a sequence of probability measures on $\mathbb{T}$.

At stage zero we have Lebesgue measure, $\mu_{0}$, on $\mathbb{T}$. At the $n$th stage $\mathbb{T}$ is divided into $4^{n}$ disjoint equal subintervals (open on the right and closed on the left) and $\mu_{n}$ is a probability measure such that on each of these subintervals it is a constant multiple of Lebesgue measure.

The intervals at the $n$th stage will be called " $n$-intervals".

Let $f_{n}$ denote the density function for $\mu_{n}$. Thus if $I$ is an $n$-interval then

$$
f_{n}(x)=\mu_{n}(I)|I|^{-1}=4^{n} \mu_{n}(I), \quad x \in I,
$$

where $|I|=4^{-n}$ is the length of $I$.

Therefore, $\mu_{n}=f_{n} \mu_{0}$. We shall arrange things so that

$$
\mu_{j}(I)=\mu_{n}(I) \text { for all } j \geq n,
$$

if $I$ is an $n$-interval.

Using this one can show that the sequence $\left\{\mu_{n}\right\}$ converges weak* to a probability measure $\mu$ on $\mathbb{T}$, that is,

$$
\int g d \mu_{n} \rightarrow \int g d \mu
$$

for all continuous functions $g$ on $\mathbb{T}(g(0)=g(1))$. Indeed, by (18) this holds for certain discontinuous $g$; namely, if $g$ is constant on each $N$-interval for some fixed $N$. But functions of this form approximate uniformly to arbitrary continuous functions.

Notation. $f_{n}(I)$ denotes the constant value of $f_{n}$ on $I$, if $I$ is an $n$-interval (or a $j$-interval for some $j>n$ ).

Rather than specifying the measures $\mu_{n}$ directly we shall specify the sequence of density functions $\left\{f_{n}\right\}$. Condition (18) is equivalent to the following condition: if $I$ is an $n$-interval, and if $I_{1}, \ldots, I_{4}$ are the four $(n+1)$-intervals into which $I$ is divided, then

$$
f_{n}(I)=\frac{1}{4}\left[f_{n+1}\left(I_{1}\right)+\cdots+f_{n+1}\left(I_{4}\right)\right] .
$$


This is just the requirement that the sequence $\left\{f_{n}\right\}$ be a martingale.

Definition. A 4-adic martingale is a sequence $\left\{h_{n}\right\}$ of real valued functions on $\mathbb{T}$ such that $h_{n}$ is constant on each $n$-interval, and (19) is satisfied, $n=$ $0,1 \ldots$. If each $h_{n}$ is nonnegative then the martingale is said to be nonnegative.

The following result describes the properties of the density functions $f_{n}$ that we need for the proof of the existence of the measure $\mu$. This result is the main ingredient in the proof of the theorem. Before proving it we first use it to complete the proof of the theorem.

Proposition 2. Let $\psi$ and $\varphi$ be positive continuous real valued functions defined for $t \geq 0$, with $\psi$ increasing and $\varphi$ decreasing, $\psi(0) \geq 2$ and $\varphi(0) \leq 1$, $\psi(t) \rightarrow \infty$ and $\varphi(t) \rightarrow 0$ as $t \rightarrow \infty$. Assume further that

(a) $\varphi(n) \leq 2 \varphi(n+1), n=0,1,2, \ldots$;

(b) $\sum \varphi(n)^{2}=\infty$.

Then there exists a nonnegative 4-adic martingale $\left\{f_{n}\right\}$ such that

(i) $f_{0}=1$,

(ii) $\varphi(n) \leq f_{n}(x) \leq \psi(n)$ for $n \geq 0$ and $0 \leq x \leq 1$,

(iii) $\left|f_{n}(I)-f_{n}\left(I^{\prime}\right)\right| \leq \varphi(n)$ for $n \geq 0$, whenever $I$ and $I^{\prime}$ are any two adjacent $n$-intervals,

(iv) $\left|f_{n+1}(I)-f_{n}(J)\right| \leq \frac{1}{2} \varphi(n+1)$, whenever $J i$ an n-interval, and $I \subset J$ is an $(n+1)$-interval

(v) $f_{n}(x) \rightarrow 0$ almost everywhere.

We now use this result to complete the proof of Theorem 4. Let $\psi(t)=$ $\alpha\left(4^{-t}\right)$ and $\varphi(t)=\beta\left(4^{-t}\right)$, where $\alpha$ and $\beta$ are given from the hypotheses of Theorem 4. Clearly $\psi$ is increasing, $\varphi$ is decreasing, $\psi(0) \geq 2, \varphi(0) \leq 1$, and $\psi(t) \rightarrow \infty, \varphi(t) \rightarrow 0$ as $t \rightarrow \infty$. Also,

$$
\varphi(n)=\beta\left(4^{-n}\right)=\beta\left(4 \cdot 4^{-(n+1)}\right) \leq 2 \beta\left(4^{-(n+1)}\right)=2 \varphi(n+1),
$$

and so hypothesis (a) of Proposition 4 is satisfied. To see that hypothesis (b) is satisfied we proceed as follows:

$$
\begin{aligned}
\sum_{0}^{\infty} \varphi(n)^{2} & \geq \int_{0}^{\infty} \varphi(t)^{2} d t=\int_{0}^{\infty} \beta\left(4^{-t}\right)^{2} d t \\
& =(\log 4)^{-1} \int_{0}^{1} \beta(s)^{2} s^{-1} d s=\infty,
\end{aligned}
$$

where the last equality is given by hypothesis (b) of Theorem 4 . Thus we have verified all the hypotheses of the proposition. We now apply the proposition to obtain the existence of the functions $\left\{f_{n}\right\}$.

Let $\mu_{n}=f_{n} \mu_{0}$ (as before, $\mu_{0}$ denotes Lebesgue measure on $\mathbb{T}$ ); then the $\left\{\mu_{n}\right\}$ are probability measures on $\mathbb{T}$ satisfying (18), and so they converge weak* to a probability measure $\mu$. Thus,

$$
\mu(I)=\mu_{j}(I)=|I| f_{n}(I)=4^{-n} f_{n}(I)
$$

for all $j \geq n$ and all $n$-intervals $I$. 
We first show that $\mu$ is a singular measure. Indeed, since $f_{n} \rightarrow 0$ a.e. there are, by Egorov's Theorem, sets $E_{k}$ with $\mu_{0}\left(E_{k}\right) \geq(k-1) / k$, so that $f_{n} \rightarrow 0$ uniformly on $E_{k}$. It follows that $\mu\left(E_{k}\right)=0$ for each $k$, and thus $\mu$ is singular.

Next we establish conclusion (1) of Theorem 4: $\omega_{1}(t ; \mu) \leq 8 t \alpha(t)$. Let $J$ be a subinterval of $\mathbb{T}$ and choose the nonnegative integer $n$ so that

$$
4^{-(n+1)}<|J| \leq 4^{-n} .
$$

Then there are two adjacent $n$-intervals $I, I^{\prime}$ such that $J \subset I \cup I^{\prime}$. (If $J$ is contained in a single $n$-interval, this will improve the inequality below by a factor of 2.) Thus

$$
\mu(J) \leq \mu(I)+\mu\left(I^{\prime}\right)=|I|\left\{f_{n}(I)+f_{n}\left(I^{\prime}\right)\right\} \leq 8|J| \psi(n),
$$

since $|I|<4|J|$. From (21) we have $n \leq \log _{4}|J|^{-1}$. Therefore,

$$
\mu(J) \leq 8|J| \psi\left(\log _{4}|J|^{-1}\right)=8|J| \alpha(|J|),
$$

and conclusion (1) of the theorem follows from this. In particular, this implies that a single point has $\mu$-measure zero.

Finally we establish conclusion (2) of Theorem $4: \omega_{2}(t ; \mu) \leq 36 t \beta(t)$. To do this we must show that if $J, J^{\prime}$ are two adjacent subarcs of $\mathbb{T}$ of the same length, then

$$
\left|\mu(J)-\mu\left(J^{\prime}\right)\right| \leq 36|J| \beta(|J|) .
$$

It is sufficient to prove this for open subarcs, since points have measure zero. Let $J$ and $J^{\prime}$ be given, and choose $n$ so that (21) is satisfied. We work first with $J$.

There are at most three $(n+1)$-intervals contained in $J$ (since each such interval contains its left endpoint, whereas $J$ is an open arc). The union of these intervals is a subinterval of $J$ (or is empty).

Let $\sum I_{n+1}$ denote the union of all the $(n+1)$-intervals (if any) contained in $J$; there are at most three such intervals.

Let $\sum I_{n+2}$ denote the union of all the $(n+2)$-intervals contained in $J \backslash \sum I_{n+1}$; there are at most six such intervals (consider separately the two cases that $J$ does, or does not, contain at least one $(n+1)$-interval).

Let $\sum I_{n+3}$ denote the union of all the $(n+3)$-intervals contained in $\left(J \backslash \sum I_{n+1}\right) \backslash \sum I_{n+2}$; there are at most six such intervals.

Continuing in this manner we have a decomposition of $J$. In the next few paragraphs we shall use the notation $I_{j}(j \geq n+1)$ to denote the $j$-intervals in this decomposition of $J$. Thus we have

$$
\left(\begin{array}{rl}
|J| & =\sum\left|I_{n+1}\right|+\sum\left|I_{n+2}\right|+\sum\left|I_{n+3}\right|+\cdots, \\
\mu(J) & =\sum \mu\left(I_{n+1}\right)+\sum \mu\left(I_{n+2}\right)+\sum \mu\left(I_{n+3}\right)+\cdots .
\end{array}\right.
$$

There are two adjacent $n$-intervals $I, I^{\prime}$ such that $J \subset I \cup I^{\prime}$. (If $J$ lies in a single $n$-interval then the term $\varphi(n)$ on the right side of each of the estimates 
below can be deleted.) Thus each interval $I_{n+1}, I_{n+2}, \ldots$ lies either in $I$ or in $I^{\prime}$.

Notation. If $H$ is a $j$-interval, then $D(H)$ will denote $f_{j}(H)$.

If $I_{n+1} \subset I$, then $\left|D\left(I_{n+1}\right)-D(I)\right| \leq \frac{1}{2} \varphi(n+1)$, by (iv) of Proposition 2. On the other hand if $I_{n+1}$ is contained in $I^{\prime}$, then

$$
\left|D\left(I_{n+1}\right)-D(I)\right| \leq \frac{1}{2} \varphi(n+1)+\varphi(n) \leq \frac{3}{2} \varphi(n),
$$

by (iii) and (iv) of Proposition 2.

If $I_{n+2} \subset I$, then $\left|D\left(I_{n+2}\right)-D(I)\right| \leq \frac{1}{2}\{\varphi(n+2)+\varphi(n+1)\}$, whereas if $I_{n+2} \subset I^{\prime}$, then

$$
\left|D\left(I_{n+2}\right)-D(I)\right| \leq \frac{1}{2}\{\varphi(n+2)+\varphi(n+1)\}+\varphi(n) \leq \frac{4}{2} \varphi(n) .
$$

Thus in general we have

$$
2\left|D\left(I_{n+k}\right)-D(I)\right| \leq(k+2) \varphi(n) .
$$

Combining this with (23) we have

$$
\begin{aligned}
|\mu(J)-| J|D(I)| \leq & \sum\left|I_{n+1}\right|\left|D\left(I_{n+1}\right)-D(I)\right| \\
& +\sum\left|I_{n+2}\right|\left|D\left(I_{n+2}\right)-D(I)\right|+\cdots \\
\leq & 4^{-n-1} \varphi(n)\left(3 \cdot \frac{3}{2}+3 \sum_{1}^{\infty}(k+3) 4^{-k}\right)<9|J| \varphi(n) .
\end{aligned}
$$

Together with the corresponding inequality for $J^{\prime}$ we have

$$
\left|\mu(J)-\mu\left(J^{\prime}\right)\right| \leq 18|J| \varphi(n) .
$$

Finally, from (21) and the fact that $\varphi$ is decreasing we have

$$
\varphi(n) \leq 2 \varphi(n+1) \leq 2 \varphi\left(-\log _{4}|J|\right) \leq 2 \beta(|J|) .
$$

This establishes conclusion (2) of Theorem 4. Thus it remains to prove the proposition.

\section{Proof of Proposition 2}

Notations. 1. $\tau(n)=\varphi(n)-\varphi(n+1)$. Thus, $\tau(n) \leq \frac{1}{2} \varphi(n) \leq \varphi(n+1)$.

2. $\tilde{\psi}=\psi-\varphi$. Thus $\tilde{\psi}$ is an increasing function, and $\tilde{\psi}(0) \geq 1$.

3. $d_{n}=f_{n}-f_{n-1}(n \geq 1)$. Thus $d_{n}$ is constant on each $n$-interval.

4. $s_{n}\left(I, I^{\prime}\right)=\operatorname{signum}\left\{f_{n}(I)-f_{n}\left(I^{\prime}\right)\right\}$, when $I, I^{\prime}$ are adjacent $n$-intervals. We take $\operatorname{sgn}(0)=1$.

5. $|\{f<b\}|$ denotes the Lebesgue measure of the set of points $x$ in $[0,1]$ for which $f(x)<b$.

Let $f_{0}=1$, and $p_{0}=0$. Note that $f_{0} \leq \tilde{\psi}(0)$. We shall construct inductively a sequence of nonnegative functions $\left\{f_{n}\right\}$, and a strictly increasing sequence of 
integers $\left\{p_{k}\right\}$, such that the following five conditions are satisfied:

$$
\begin{gathered}
f_{n-1}(I)=\frac{1}{4}\left[f_{n}\left(I_{1}\right)+\cdots+f_{n}\left(I_{4}\right)\right], \quad(n \geq 1) ; \\
\varphi(n) \leq f_{n} \leq \tilde{\psi}(n) ; \\
\left|f_{n}(I)-f_{n}\left(I^{\prime}\right)\right| \leq \varphi(n) \quad\left(I, I^{\prime} \text { are adjacent } n \text {-intervals }\right) ; \\
\left|f_{n}(I)-f_{n-1}(J)\right| \leq \frac{1}{2} \varphi(n) \quad(J \text { in as }(n-1) \text {-interval, and } \\
I \subset J \text { is an } n \text {-interval; } n \geq 1) ; \\
\left|\left\{f_{p_{i}} \leq \varphi\left(p_{i-1}\right)\right\}\right| \geq i /(i+1) \quad(i \geq 1) .
\end{gathered}
$$

This construction will prove Proposition 2. Indeed, only conclusion (v): $f_{n}(x) \rightarrow 0$ a.e., needs to be verified. By passing to a subsequence of the sequence $\left\{p_{i}\right\}$ we may obtain a subsequence of $\left\{f_{n}\right\}$, which we label $\left\{f_{n_{k}}\right\}$, such that if

$$
E_{k}=\left\{x: f_{n_{k}}(x) \leq 1 / k\right\}
$$

then $\left|E_{k}\right| \geq 1-k^{-2}$. Let $E$ denote the set of points $x$ lying in all but finitely many of the sets $E_{k}$. Using the fact that $\sum\left|E_{k}^{c}\right|<\infty$, where $E_{k}^{c}=\mathbb{T} \backslash E_{k}$, one shows that $|E|=1$ (the Borel-Cantelli lemma). Thus

$$
\lim _{k} f_{n_{k}}(x)=0 \text { almost everywhere. }
$$

But $\left\{f_{n}\right\}$ is a positive martingale and therefore converges almost everywhere (see [Db53, Chapter VII, Theorem 4.1, p. 319]), which completes the proof of (v).

For $n=i=0,(24),(27)$, and (28) are vacuously satisfied, (26) is trivial, the upper bound in $(25)$ was indicated above, and the lower bound comes because $\varphi(0) \leq 1$.

Induction Assumption. The integers $p_{0}, \ldots, p_{k}$ and the functions $f_{0}, \ldots, f_{n}$ have been chosen such that for all $n \leq p_{k}$ and all $i \leq k$, conditions (24)-(28) are satisfied.

In every case, $f_{n+1}$ will be obtained by increasing and decreasing $f_{n}$ symmetrically, by either $\tau(n) / 2$ or $\varphi(n+1) / 2$, so that (24) and (27) are both satisfied. Thus in what follows we shall not discuss these two conditions.

We first choose an intermediate integer $r_{k}$ and define $f_{n+1}$ for $p_{k} \leq n<r_{k}$ so that (24)-(27) are satisfied; then we show how to define $f_{n}$ for $n>r_{k}$, and finally we choose $p_{k+1}$ so that all five conditions are satisfied. This will complete the induction.

Let $N$ be the smallest positive integer such that $N>(k+2) \psi\left(p_{k}\right)$. This is equivalent to

$$
N-\psi\left(p_{k}\right)>N(k+1) /(k+2) .
$$

Now let $r_{k}$ be the smallest integer greater than $p_{k}$ such that

$$
5 \varphi\left(r_{k}\right)<2 \varphi\left(p_{k}\right), \quad \check{\psi}\left(r_{k}\right)>N+1 .
$$


For $p_{k} \leq n<r_{k}$ we define $d_{n+1}$ recursively as follows. Let $I$ be an $n$ interval, let $I_{1}, \ldots, I_{4}$ be the four $(n+1)$-intervals contained in $I$, and let $I^{\prime}$ and $I^{\prime \prime}$ be the $n$-intervals to the left and to the right of $I$ (recall that 0 and 1 are identified.). Note that by $(25) f_{n}(I)>0$. Then

$$
d_{n+1}\left(I_{1}\right)=s_{n}\left(I^{\prime}, I\right) \tau(n) / 2, \quad d_{n+1}\left(I_{4}\right)=s_{n}\left(I^{\prime \prime}, I\right) \tau(n) / 2,
$$

and $d_{n+1}\left(I_{2}\right)=-d_{n+1}\left(I_{1}\right), d_{n+1}\left(I_{3}\right)=-d_{n+1}\left(I_{4}\right)$. Let $f_{n+1}=f_{n}+d_{n+1}$. Since $\left|d_{n+1}\right| \leq \tau(n)$ we have

$$
\begin{aligned}
& f_{n+1} \leq \tilde{\psi}(n)+\tau(n)=\psi(n)-\varphi(n+1) \leq \tilde{\psi}(n+1), \\
& f_{n+1} \geq \varphi(n)-\tau(n)=\varphi(n+1) .
\end{aligned}
$$

Thus $f_{n+1}$ satisfies condition (25). As regards (26) we have

$$
\left|f_{n+1}\left(I_{j}\right)-f_{n+1}\left(I_{j+1}\right)\right| \leq \tau(n) \leq \varphi(n+1), \quad j=1,2,3 .
$$

Let $I_{4}^{\prime}$ denote the rightmost $(n+1)$-interval in $I^{\prime}$, and $I_{1}^{\prime \prime}$ the leftmost interval in $I^{\prime \prime}$. By $(26) f_{n}(I)$ and $f_{n}\left(I^{\prime}\right)$ differ by at most $\varphi(n)$. We distinguish two cases: (1) the difference is at least $\tau(n)$, and (2) the difference is less than $\tau(n)$. In the first case when we pass to $f_{n+1}$ the difference is reduced by $\tau(n)$ and we have

$$
\left|f_{n+1}\left(I_{1}\right)-f_{n+1}\left(I_{4}^{\prime}\right)\right| \leq \varphi(n)-\tau(n)=\varphi(n+1) .
$$

In the second case we have $\left|f_{n+1}\left(I_{1}\right)-f_{n+1}\left(I_{4}^{\prime}\right)\right| \leq \tau(n) \leq \varphi(n+1)$. One has similar estimates for $I_{4}$ and $I_{1}^{\prime \prime}$, and so $f_{n+1}$ satisfies (26).

Thus $f_{n}$ has been defined for $p_{k}<n \leq r_{k}$ and the four conditions (24)-(27) are satisfied. Before proceeding we need an additional estimate. For $p_{k}<n \leq$ $r_{k}$ we have (by dropping the factor $\frac{1}{2}$ from (31)),

$$
\left|f_{n}-f_{p_{k}}\right| \leq\left|d_{1+p_{k}}\right|+\cdots+\left|d_{n}\right| \leq \varphi\left(p_{k}\right)-\varphi(n) .
$$

Thus from (25) we have $f_{n} \leq \tilde{\psi}\left(p_{k}\right)+\varphi\left(p_{k}\right)-\varphi(n)<\psi\left(p_{k}\right)$. From this and the lower bound in (25) we have, for $n=r_{k}$,

$$
\varphi\left(r_{k}\right) \leq f_{r_{k}} \leq \psi\left(p_{k}\right) .
$$

We shall next make a preliminary definition of $f_{n}$ for all $n>r_{k}$, so that conditions (24)-(27) are satisfied. Then we show how to choose $p_{k+1}$ so that condition (28) is satisfied. Finally, we discard the $f_{n}, n>p_{k+1}$, given by the preliminary definition, thereby completing the induction.

We assume that $f_{n}$ has already been defined for some $n \geq r_{k}$; we now define $d_{n+1}$. Let $I$ be an $n$-interval, with adjacent intervals $I^{\prime}$ and $I^{\prime \prime}$ on the left and right. We distinguish two cases (A) and (B).

(A) We have $2 \varphi\left(r_{k}\right) \leq f_{i}(I) \leq N$ for $r_{k} \leq i \leq n$. Then we define

$$
d_{n+1}\left(I_{1}\right)=s_{n}\left(I^{\prime}, I\right) \varphi(n+1) / 2, \quad d_{n+1}\left(I_{4}\right)=s_{n}\left(I^{\prime \prime}, I\right) \varphi(n+1) / 2,
$$


and $d_{n+1}\left(I_{2}\right)=-d_{n+1}\left(I_{1}\right), d_{n+1}\left(I_{3}\right)=-d_{n+1}\left(I_{4}\right)$. Let $f_{n+1}=f_{n}+d_{n+1}$. Then for $i \leq j \leq 4$ we have, from $(30)$,

$$
\begin{aligned}
& f_{n+1}\left(I_{j}\right) \leq f_{n}(I)+\varphi(n+1) \leq N+\varphi\left(r_{k}\right) \leq \tilde{\psi}\left(r_{k}\right) \leq \tilde{\psi}(n+1), \\
& f_{n+1}\left(I_{j}\right) \geq f_{n}(I)-\varphi(n+1) \geq 2 \varphi\left(r_{k}\right)-\varphi(n+1) \geq \varphi(n+1) .
\end{aligned}
$$

Thus $f_{n+1}$ satisfies condition (25) on those intervals $I$ covered by (A). As regards $(26)$, for the moment we merely note that

$$
\left|f_{n+1}\left(I_{j}\right)-f_{n+1}\left(I_{j+1}\right)\right| \leq \varphi(n+1), \quad j=1,2,3 .
$$

(B) Either

(a) $f_{i}(I)<2 \varphi\left(r_{k}\right)$ for some $i, r_{k} \leq i \leq n$, or

(b) $f_{i}(I)>N$ for some $i, r_{k} \leq i \leq n$.

In both cases (a) and (b) we define

$$
d_{n+1}\left(I_{1}\right)=s_{n}\left(I^{\prime}, I\right) \tau(n) / 2, \quad d_{n+1}\left(I_{4}\right)=s_{n}\left(I^{\prime \prime}, I\right) \tau(n) / 2,
$$

and $d_{n+1}\left(I_{2}\right)=-d_{n+1}\left(I_{1}\right), d_{n+1}\left(I_{3}\right)=-d_{n+1}\left(I_{4}\right)$. Let $f_{n+1}=f_{n}+d_{n+1}$. Note that once (a) or (b) has occurred, say at index $i$, then we are in (B) for $i \leq j \leq$ $n$. Thus

$$
\left|f_{n+1}(x)-f_{i}(x)\right|=\frac{1}{2}\{\tau(i)+\cdots+\tau(n)\}=\frac{1}{2}\{\varphi(i)-\varphi(n+1)\} .
$$

We first consider (a). Let $x$ be given with $\varphi\left(r_{k}\right) \leq f_{m}(x) \leq N, r_{k} \leq m<i$, whereas $f_{i}(x)<2 \varphi\left(r_{k}\right)$. Then $f_{i}(x)$ is determined by $(\mathrm{A})$, and so

$$
2 \varphi\left(r_{k}\right)-\frac{1}{2} \varphi(i) \leq f_{i}(x)<2 \varphi\left(r_{k}\right) .
$$

Then from (37) we have

$$
\begin{aligned}
f_{n+1}(x) & \geq f_{i}(x)-\frac{1}{2}\{\varphi(i)-\varphi(n+1)\} \\
& \geq 2 \varphi\left(r_{k}\right)-\varphi(i)+\frac{1}{2} \varphi(n+1) \geq \frac{3}{2} \varphi(n+1) .
\end{aligned}
$$

and

$$
\begin{aligned}
f_{n+1}(x) & <2 \varphi\left(r_{k}\right)+\frac{1}{2}\{\varphi(i)-\varphi(n+1)\}<\frac{5}{2} \varphi\left(r_{k}\right) \\
& <\varphi\left(p_{k}\right)<\tilde{\psi}(n+1) .
\end{aligned}
$$

The last two inequalities come from (3) and the fact that $\varphi \leq 1 \leq \tilde{\psi}$.

We next consider (b). Suppose that $2 \varphi\left(r_{k}\right) \leq f_{m}(x) \leq N, r_{k} \leq m<i$, and that $f_{i}(x)>N$. Then $f_{i}(x)$ is determined by $(\mathrm{A})$, and so

$$
N<f_{i}(x) \leq N+\frac{1}{2} \varphi(i) .
$$

From this, as above, we obtain from (37), (30), and (29)

$$
\begin{aligned}
& f_{n+1}(x) \leq N+\varphi(i)-\frac{1}{2} \varphi(n+1)<N+\varphi\left(r_{k}\right)<\tilde{\psi}\left(r_{k}\right) \leq \tilde{\psi}(n+1), \\
& f_{n+1}(x)>N-\frac{1}{2}\{\varphi(i)-\varphi(n+1)\}>N-\frac{1}{2} \geq \varphi(n+1) .
\end{aligned}
$$

Thus in every case $f_{n+1}$ satisfies condition (25). 
As regards (26), we first note that $(35)$ is valid in case $(\mathrm{B})$, Since $\tau(n) \leq$ $\varphi(n+1)$. Suppose now that $I$ is an $n$-interval, and $I^{\prime}, I^{\prime \prime}$ are the $n$-intervals to the left and right of $I$. We shall show that

$$
\left|f_{n+1}\left(I_{1}\right)-f_{n+1}\left(I_{4}^{\prime}\right)\right| \leq \varphi(n+1)
$$

(the proof for $I_{4}, I_{1}^{\prime \prime}$ is similar). If both $f_{n+1}\left(I_{1}\right)$ and $f_{n+1}\left(I_{4}^{\prime}\right)$ are determined by (B), then (39) is proved as in the discussion following (31). Next assume that both are determined by $(\mathrm{A})$. By the induction hypothesis, $f_{n}(I)$ and $f_{n}\left(I^{\prime}\right)$ differ by at most $\varphi(n)$. We distinguish two cases: (1) the difference is at least $\varphi(n+1)$, and (2) the difference is less than $\varphi(n+1)$. In the first case when we pass to $f_{n+1}$ the difference is reduced by $\varphi(n+1)$ and we have

$$
\left|f_{n+1}\left(I_{1}\right)-f_{n+1}\left(I_{4}^{\prime}\right)\right| \leq \varphi(n)-\varphi(n+1) \leq \varphi(n+1) .
$$

In the second case we have $\left|f_{n+1}\left(I_{1}\right)-f_{n+1}\left(I_{4}^{\prime}\right)\right| \leq \varphi(n+1)$. Finally, we assume that (A) and (B) are both involved, say $f_{n+1}\left(I_{1}\right)$ is determined by $(\mathrm{A})$, and $f_{n+1}\left(I_{4}^{\prime}\right)$ by $(\mathrm{B})$. As before $f_{n}(I)$ and $f_{n}\left(I^{\prime}\right)$ differ by at most $\varphi(n)$. We again distinguish two cases: (1) the difference is at least $\frac{1}{2}\{\tau(n)+\varphi(n+1)\}=\varphi(n) / 2$, and (2) the difference is less than $\varphi(n) / 2$. In the first case when we pass to $f_{n+1}$ the difference is reduced by $\varphi(n) / 2$ and we have

$$
\left|f_{n+1}\left(I_{1}\right)-f_{n+1}\left(I_{4}^{\prime}\right)\right| \leq \varphi(n)-\frac{1}{2} \varphi(n) \leq \varphi(n+1) .
$$

In the second case we have $\left|f_{n+1}\left(I_{1}\right)-f_{n+1}\left(I_{4}^{\prime}\right)\right| \leq \frac{1}{2} \varphi(n) \leq \varphi(n+1)$. Thus (39) has been proved and so $f_{n+1}$ satisfies (26). This completes the preliminary definition of $f_{n}$ promised earlier for all $n>r_{k}$.

Our next task is to choose $p_{k+1}$, larger than $r_{k}$, such that (28) is satisfied for $k+1$, that is, such that

$$
\left|\left\{f_{p_{k+1}} \leq \varphi\left(p_{k}\right)\right\}\right| \geq(k+1) /(k+2) .
$$

By $(25), f_{r_{k}} \geq \varphi\left(r_{k}\right)$. Let

$$
E=\left\{x: f_{r_{k}}(x)<2 \varphi\left(r_{k}\right)\right\},
$$

and let $F=[0,1] \backslash E=\left\{f_{r_{k}} \geq 2 \varphi\left(r_{k}\right)\right\}$. Also, let

$$
D=\left\{x: 2 \varphi\left(r_{k}\right) \leq f_{n}(x) \leq N, r_{k} \leq n<\infty\right\}
$$

Then $D \subset F$. On $D$ case (A) always applies, and thus

$$
\sum d_{n}^{2}=\sum \varphi(n)^{2}=\infty
$$

by hypothesis (b) of Proposition 2. Thus the sequence of functions $\left\{f_{n}\right\}, r_{k} \leq$ $n<\infty$, is a martingale whose square function, $S=\sum d_{n}^{2},\left(n \geq r_{k}\right)$, is infinite on the set $D$. By a theorem of Burkholder (see [Bu73, Theorem 3.1, p. 21]), the square function of a positive martingale (more generally, of an $L^{1}$ bounded martingale) is infinite at most on a set of measure 0, and so $|D|=0$. Therefore,

$$
|F|=\left|\left(F \cap \bigcup_{r_{k}}^{\infty}\left\{f_{i}<2 \varphi\left(r_{k}\right)\right\}\right) \cup\left(F \cap \bigcup_{r_{k}}^{\infty}\left\{f_{i}>N\right\}\right)\right| .
$$


For $x \in F \backslash D$, let $\tau(x)$ denote the first integer $i \geq r_{k}$ such that either $f_{i}(x)<2 \varphi\left(r_{k}\right)$, or $f_{i}(x)>N$. Let $A$ denote the set where the first inequality occurs, and $B$ the set where the second occurs. Then $A$ and $B$ are disjoint, and by (41): $|F|=|A \cup B|$.

Claim. $|A \cup E|>(k+1) /(k+2)$.

Let us accept this for the moment. Note that $A \cup E$ is the set of those points $x$ for which there exists an integer $i=i(x) \geq r_{k}$ with (i) $f_{i}(x)<2 \varphi\left(r_{k}\right)$, and (ii) $2 \varphi\left(r_{k}\right) \leq f_{m}(x) \leq N, r_{k} \leq m<i$. Thus for all $n>i(x), f_{n}(x)$ is determined by case (B) above. By (38) we have:

$$
f_{n}(x)<\frac{5}{2} \varphi\left(r_{k}\right), \quad i(x)<n<\infty .
$$

Thus

$$
A \cup E \subset \bigcup_{1}^{\infty} G_{n},
$$

where $G_{n}=\left\{x: f_{j}(x)<\frac{5}{2} \varphi\left(r_{k}\right), n \leq j<\infty\right\}$. Since this is an increasing union the above claim implies that $\left|G_{n}\right|>(k+1) /(k+2)$ for all sufficiently large $n$. Let $p_{k+1}$ be the first such integer $n$ larger than $p_{k}$. Since $\frac{5}{2} \varphi\left(r_{k}\right)<\varphi\left(p_{k}\right)$ by (30), we see that with this choice of $p_{k+1}$ inequality (40) will be valid. This completes the induction.

It remains to establish the above claim. This is trivial if $|F|=0$, so we assume that $F$ has positive measure. We require the following result.

Lemma 5. Let $\left\{f_{n}\right\}, M \leq n<\infty$, be a martingale on a probability measure space with measure $P$. Let $\varepsilon, a, b, c, d$ be the real numbers with $\varepsilon>0$, and $a \leq c<d \leq b$. Assume that

(a) $c \leq f_{M} \leq d$ a.e.;

(b) $\left|f_{n+1}-f_{n}\right| \leq \varepsilon, M \leq n<\infty$;

(c) $P\left(a \leq f_{n} \leq b, M \leq n<\infty\right)=0$.

Let

$$
\tau_{a}(x)=\inf \left\{k: f_{k}(x)<a\right\}, \quad \tau_{b}(x)=\inf \left\{k: f_{k}(x)>b\right\},
$$

and let $t=\int f_{M}$. Then

$$
\frac{b-t}{b-a+\varepsilon} \leq P\left(\tau_{a}<\tau_{b}\right) \leq \frac{b+\varepsilon-t}{b-a+\varepsilon} .
$$

Proof. Let $\tau=\min \left(\tau_{a}, \tau_{b}\right)$. Hypothesis (c) says that $\tau(x)<\infty$ almost everywhere. Let $A$ (resp. B) denote the set where $\tau<\infty$ and $\tau=\tau_{a}$ (resp. $\left.\tau=\tau_{b}\right)$. Then $A$ and $B$ are disjoint and $P(A \cup B)=1$. By the optional stopping theorem of Doob (see [Db53, Chapter VII, Theorem 2.1, p, 300]) we have

$$
\int_{A} f_{\tau_{a}}+\int_{B} f_{\tau_{b}}=\int f_{\tau(x)}(x) d P(x)=\int f_{M} d P=t .
$$

Note that $P(A)=P\left(\tau_{a}<\tau_{b}\right)$. The result now follows from the inequalities:

$$
a-\varepsilon \leq f_{\tau_{a}}(x) \leq a, \quad x \in A ; \quad b \leq f_{\tau_{b}}(x) \leq b+\varepsilon, \quad x \in B,
$$

and the fact that $P(B)=1-P(A)$. 
We apply this result to the sequence of functions $\left\{f_{n}\right\}, r_{k} \leq n<\infty$, restricted to the set $F$. The probability measure is Lebesgue measure divided by the measure of $F$. We let $M=r_{k}, a=c=2 \varphi\left(r_{k}\right), d=\psi\left(p_{k}\right), b=N$, and $\varepsilon=\varphi\left(r_{k}\right) / 2$. The definition of the set $F$, together with (32), tells us that hypothesis (a) is fulfilled. Hypothesis (b) is clear from examining cases (A) and (B). Finally, (c) is satisfied because the set $D$ has measure zero (see the discussion preceding equation (41)). We apply the left side of (43). Since $t \leq \psi\left(p_{k}\right)$ we have, using (29),

$$
b-t=N-t \geq N-\psi\left(p_{k}\right)>N(k+1) /(k+2) .
$$

For the denominator we have the estimate

$$
b-a+\varepsilon=N-2 \varphi\left(r_{k}\right)+\varphi\left(r_{k}\right) / 2<N .
$$

From (43) we have $|A|>|F|(k+1) /(k+2)$. Since $E$ is the complement of $F$ we have $|A \cup E|>(k+1) /(k+2)$, thus establishing the claim.

This completes the proof of Proposition 2, and hence of Theorem 4.

Note added in proof. Professor Allen L. Shields died on 16th September 1989 after a painful illness courageously borne. With his death the international Mathematical Community loses an outstanding member and lucid expositor, while many mourn the loss of a personal friend. The surviving authors sadly dedicate this paper to his memory.

"His life was gentle, and the elements so mixed in him that Nature might stand up and say to all the world-This was a man!"

\section{BIBLIOGRAPHY}

[An85] J. M. Anderson, Bloch functions: the basic theory, Operators and Function Theory (S. C. Power, ed.), Reidel, Dordrecht, 1985, pp. 1-17. MR 87h:30072

[ABB77] J. M. Anderson, K. Barth, and D. Brannan, Research problems in complex analysis, Bull. London Math. Soc. 9 (1977), 129-162. MR 55 \#12899

[ACP74] J. M. Anderson, J. Clunie, and C. Pommerenke, On Bloch functions and normal functions, J. Reine Angew. Math. 20 (1974), 12-37. MR 50 \#13536

[Be49] S. N. Bernstein, On majorants of finite or quasi-finite growth, Dokl. Akad. Nauk SSSR 65 (1949), 117-120. (Russian) MR 11, 23

[BS84] L. Brown and A. L. Shields, Cyclic vectors in the Dirichlet space, Trans. Amer. Math. Soc. 285 (1984), 269-304. MR 86d:30079

[Bu73] D. L. Burkholder, Distribution function inequalities for martingales, Ann. Probab. 1 (1973), 19-42. MR 51 \#1944

[Db53] J. L. Doob, Statistic processes, Wiley, New York, 1953. MR 15, 445

[DSS66] P. L. Duren, H. S. Shapiro, and A. L. Shields, Singular measures and domains not of Smirnov type, Duke Math. J. 33 (1966), 247-254. MR 33 \#7506

[Hk37] P. Hartman and R. Kerschner, The structure of monotone functions, Amer. J. Math. 59 (1937), 809-822.

[Ka69] J.-P. Kahane, Trois notes sur les ensembles parfaits linéaires, Enseign. Math. 15 (1969), 185-192. MR $39 \# 7040$ 
[Ko81] B. I. Korenblum, Cyclic elements in some spaces of analytic functions, Bull. Amer. Math. Soc. (N.S.) 5 (1981), 317-318. MR 82j:30074

[PSW67] G. Piranian, A. L. Shields, and J. H. Wells, Bounded analytic functions and absolutely continuous measures, Proc. Amer. Math. Soc. 18 (1967), 818-826. MR 35 \#5911

[R85] J. W. Roberts, Cyclic inner functions in Bergman spaces and weak outer functions in $H^{p}$, $0<p<1$, Illinois J. Math. 29 (1985), 25-38. MR 86c:30069

[RS66] L. A. Rubel and A. L. Shields, The space of bounded analytic functions on a region, Ann. Inst. Fourier (Grenoble) 16 (1966), 235-277. RM 33 \#6440

[RS70] _ The second duals of certain spaces of analytic functions, J. Austral. Math. Soc. 11 (1970), 276-280. MR 43 \#2484

[hS64] H. S. Shapiro, Weakly invertible elements in certain function spaces and generators in $l^{1}$, Michigan Math. J. 11 (1964), 161-165. MR 29 \#3620

[hS68] __, Monotonic singular functions of high smoothness, Michigan Math. J. 15 (1968), 265275. MR $38 \# 1219$

[jS80] Joel Shapiro, Cyclic inner functions in Bergman spaces, preprint, 1980 (not for publication).

[S85] A. L. Shields, Cyclic vectors in Banach spaces of analytic functions, Operators and Function Theory (S. C. Power, ed.), Reidel, Dordrecht, 1985, pp. 315-349. MR 87c:47048

[SW78] A. L. Shields and D. L. Williams, Bounded projections, duality, and multipliers in spaces of harmonic functions, J. Reine Angew. Math. 299/300 (1978), 256-279. MR 58 \#7053

[SW82] __ Bounded projections and the growth of harmonic conjugates in the unit disc, Michigan Math. J. 29 (1982), 3-25. MR 83h:30063

[SZ63] E. M. Stein and A. Zygmund, On the differentiability of functions, Studia Math. 23 $(1963 / 64), 247-283$. (Note: apparently this paper was never reviewed in the Mathematical Reviews.)

Department of Mathematics, University College London, Gower St., London WCiE 6BT, ENGLAND

Division de Matematicas, Facultad de Ciencias, Universidad Autónoma de Madrid, Ciudad Universitaria de Canto Blanco, 28049 Madrid, Spain

Department of Mathematics, University of Michigan, Ann Arbor, Michigan 481091003 